\title{
Prospects for the Application of Artificially Cultured Diatom Materials in Energy and Environment
}

\author{
Jintao Huang, Dazhi Sun * and Jaw-Kai Wang
}

Received: 25 May 2020; Accepted: 31 May 2020

Article type: Personal account

Diatoms are a kind of eukaryotic organisms, which are common single-celled algae in nature. They are various in species, large in quantity and widely distributed. ${ }^{[1,2]}$ Diatoms are very small, ranging from 0.01 to $0.1 \mathrm{~mm}$. Photosynthesis of diatom plants can absorb carbon dioxide and release oxygen, which has a large influence on global climate change. As an important biological resource, diatom is one of the main foods for fish, shell fish and other aquatic animals, and plays an important role in aquatic ecosystem and biological environment monitoring. ${ }^{[3]}$ Diatoms have special silicified cell walls (diatom frustules), which can be divided into two basic types: radiation symmetry and bilateral symmetry. Diatom frustules are unique biological inorganic materials with extremely high purity in nature and are also one of the best micro-nano biological platform materials, which have very important research significance. ${ }^{[4]}$ Diatom frustules not only enhance the hardness and strength of diatom, offering the mechanical property of its suspending, but also improve its physiological function of transporting nutrients, adsorption and adhesion, and prevent the entry of harmful substances, making it more and more widely used.

At present, almost all the diatoms are grown in nature, and the most used diatom-based material is diatomite, which is a mineral formed by diatom frustules after tens of millions of years. $^{[5,6]}$ Diatom frustules contain $98 \%$ silica, but the commercial application of diatoms is different from both diatomite and synthetic silica materials. Each individual diatom has only three components: one-third of diatom frustules, one-third of proteins, and one-third of diatom lipids. Therefore, the diatom industry would be able to solve three fundamental global problems: industrial feedstocks (diatom

SUSTech-Taili Joint Lab for Diatom Materials, Department of Materials Science and Engineering, Southern University of Science and Technology, Shenzhen, Guangdong 518055, China

*E-mail: sundz@sustech.edu.cn (D. Sun) frustules), energy (diatom lipids) and food (proteins). ${ }^{[7,8]}$ Although the entire body of diatom is useful, there are technical problems such as high culture cost, difficult separation technology, etc., which cannot be artificially cultured on a large scale. ${ }^{[9]}$ In the last century, US Renewable Energy Laboratory spent more than 20 years of research, but still only stayed in the culture under laboratory conditions, leading to the suspension of the industrialization of diatoms. In the last decade, Jaw-Kai Wang, who is a member of National Academy of Engineering (USA), finally has achieved the successful outdoor large-scale artificial culture of diatoms for the first time. ${ }^{[10]}$ Considering the characteristics of wide distribution, fast growth and strong competitive advantage of diatoms, Jaw-Kai Wang operated an experimental outdoor system to produce 120 tons of dry diatom per hectare per year.

Up to now, some substantial achievements have been made in the laboratory stage, some of which have been used in the pilot process or even industrial applications, as shown in Fig. 1 and described below. Based on the diatom frustules material, Dazhi Sun's team of SUSTech-Taili Joint Lab for Diatom Materials has developed a superhydrophobic coating with micro-nano structure on the surface, which can make the surface contact angle of the material reach $150^{\circ}$. At the same time, the coating has friction resistance, scratch resistance, corrosion resistance and so on, and can be prepared on a large scale. Superhydrophobic structural materials based on diatom frustules can be widely used in the fields of architecture, transportation, machinery, solar energy, aerospace, ocean engineering, communication engineering, etc. Based on the unique structure and performance characteristics of diatom frustules, Jaw-Kai Wang's team of Shenzhen Jawkai Bioengineering R\&D Center Co., Ltd. invented a biological silicon heavy metal adsorbent for the treatment of heavy metal wastewater. ${ }^{[1]}$ Biosilicon heavy metal adsorbent is a kind of natural silicon-based mesoporous adsorption material, 


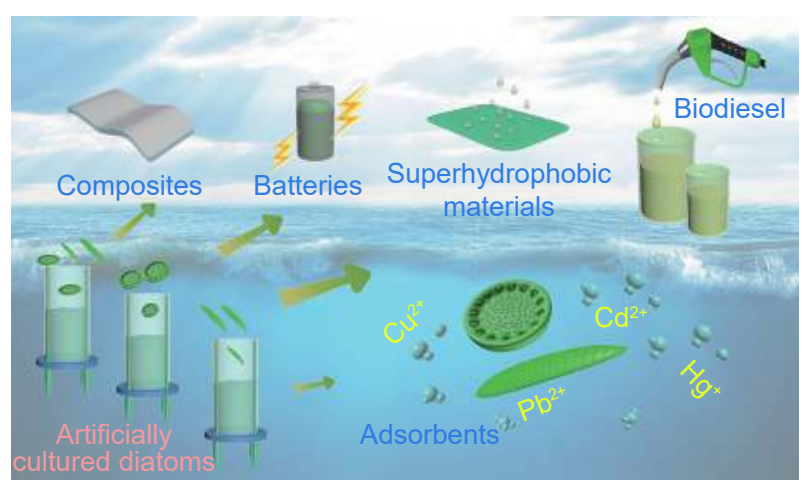

Fig. 1 Schematic diagram of artificially cultured diatom materials and their applications.

which has the characteristics of large adsorption capacity, high removal efficiency, stable property, reusable, renewable, natural environmental protection and low cost. At present, the diatom frustule adsorbent can absorb copper, mercury, cadmium, chromium, lead, silver and other pollutants that are common in water body, and can be reused more than 200 times. Wang's team also used diatom lipids as raw material to produce pure hydrocarbon bioenergy products (such as pure hydrocarbon biodiesel) through catalytic deoxidization process, and the deoxidization rate of the products can reach more than 99\%. Pure hydrocarbon biodiesel and petrochemical diesel have the same chemical composition, the same physical and chemical properties and the same power performance, which can be used in the engines. After refining and blending, the product can be seamlessly connected with the petrochemical diesel market. In addition, there are many other applications of diatom materials, such as diatom-based electrode materials for lithium-ion batteries, diatom-based phase change materials, diatom-based biodegradable plastics, diatom-based micro-nano structure stealth materials and so on.

Based on the above-mentioned stage achievements, we may know that diatom industry is an extremely large ecological industry system. It is not only a revolution in the field of materials, but also meets the needs of various highgrade, precision and advanced materials in countries around the world, and will play a huge role in industry, agriculture, national defense, medicine, environmental protection, food and energy security. Moreover, all productions in the chain of artificially cultured diatoms are completely pollution-free, which meets the environmental protection requirements of global industrial development. In addition, $80 \%$ of the diatomic-derived industries that are related to new materials and new energy are the key support for the revitalization of important industries and technological upgrade in many countries with huge development prospects. Therefore, it is expected that the diatom materials from artificial culture will have great impact on both academia and practical applications in the fields of energy and environment.

\section{Acknowledgements}

The work was supported by Southern University of Science and Technology and Taili Energy Co. Ltd. We also thank He Han and Tao Li for their discussion.

\section{Supporting information}

Not applicable

\section{Conflict of interest}

There are no conflicts to declare.

\section{References}

[1] J. Seckbach and R. Gordon, Diatoms: Fundamentals and Applications, Wiley: Scrivener Publishing, 2019.

[2] A. Csordas and J. K. Wang, Aquacult. Eng., 2004, 30, 15-30.

[3] H. Li, J. Watson, Y. Zhang, H. Lu and Z. Liu, Bioresoure Technol., 2020, 298, 122421.

[4] A. E. F. Abomohra, W. Jin, R. Tu, S. F. Han, M. Eid and H. Eladel, Renew. Sust. Energ. Rev., 2016, 64, 596-606.

[5] I. I. Nesterov, P. V. Smirnov, A. O. Konstantinov and H. J. Gursky, Int. Geol. Rev., 2020, 1-22.

[6] J. Witkowski, P. A. Sims and D. M. Williams, Diatom Res., 2017, 32, 363-408

[7] C. Baldisserotto, A. Sabia, L. Ferroni and S. Pancaldi, World J. Microb. Biot., 2019, 35, 35

[8] A. Amato, V. Sabatino, G. M. Nylund, J. Bergkvist, S. Basu, M. X. Andersson, R. Sanges, A. Godhe, T. Kiorboe, E. Selander and M. I. Ferrante, ISME J., 2018, 12, 1594-1604.

[9] J. Zhou, Y. Wu, J. Pan, Y. Zhang, Z. Liu, H. Lu and N. Duan, Sci. Total Environ., 2019, 694, 133720.

[10] J. K. Wang and M. Seibert, Biotechnol. Biofuels, 2017, 10, 16.

[11] J. Qin, J. K. Wang and J. Zhang, Guangdong Chem. Ind., 2016, 16, 93-95.

Publisher's Note: Engineered Science Publisher remains neutral with regard to jurisdictional claims in published maps and institutional affiliations. 